\title{
Fabrication of stainless steel PEMFC bipolar plate by soft punch stamping
}

\author{
Hanxia RUAN ${ }^{1, a}$, Tao $\mathrm{CHEN}^{2, b^{*}}$ \\ ${ }^{1}$ School of Material Science and Engineering, Wuhan University of Technology, CHINA \\ ${ }^{2}$ School of Mechanical and Electronic Engineering, Wuhan University of Technology, CHINA \\ arhx_wut@163.com, bchent29@163.com \\ ${ }^{*}$ Corresponding author
}

\begin{abstract}
Keywords: Bipolar plate; Flow channel; Stamping process; Finite element simulation
Abstract. Different from traditional forming process, sheet soft punch process uses only a rigid die and the other tool set is a flexible medium, such as natural or synthetic rubbers. Soft punch stamping process for flow channel in stainless steel bipolar plates of the proton exchange membrane fuel cell (PEMFC) is an economic and convenient method for mass production. In order to investigate the process of soft punch stamping, a three-dimensional finite element model of bipolar plate is developed to analyze the strain variation during the stamping process and predict the defects.
\end{abstract}

\section{Introduction}

Soft punch stamping process is an efficient way to form metal sheet with the liquid, gas or rubber medium instead of rigid punch, especially in the production of micro forming [1-4]. It saves time and cost to design the process and tools compared with the traditional sheet forming processes. To avoid the defects such as wrinkling and rupture, the accurate assembly of traditional rigid die, punch and other attachments is very complex

Soft punch stamping process only use a rigid die, and the punch is made of soft material such as natural or synthetic rubbers. So only the rigid punch need to be designed and accurately manufactured and there is no problem in the assembly of soft punch and rigid die.

Currently, the most commonly used soft material is synthetic rubbers, of which physical properties fall between plastic and rubber properties. This rubber material is widely used in machinery equipment, owing to its large range of rigidity, high strength, high elasticity and a small amount of residual deformation, good wear resistance, oil resistance, aging resistance and tear resistance. In addition, its mechanical performance is also very good.

Early in the 19th century, the synthetic rubber is widely used for good elasticity such as load-bearing component in bearings, seals, shock-absorbing pads, connectors. While at present, the most important application of synthetic rubbers is used to replace the traditional material of rigid die or punch, which can simplify the structure of the conventional die and expand the application range of punching technology, making possible the sheet stamping that is hard for traditional stamping.

In stamping process synthetic rubbers is also used as an elastic component among many researchers, such as the discharging device, the buffer and the blank holder, as well as a top clamping member in the mold and the press.

One of the application of soft punch is the manufacture of the bipolar plate in proton exchange membrane fuel cell [5]. It's an important component with many micro channel to provide conduits for reactant gases and coolant flow, and also constitutes the backbone of a fuel cell stack.

In this paper we establish a finite element analysis model to study bipolar plate forming process using soft punch.

\section{Material model for soft punch}

The soft punch material is a kind of rubber, which is generally considered to be isotropic incompressible and hyper-elastic model(Moone-Rivlin) is used to describe its behavior. The 
Mooney-Rivlin model adds a term that depends on the second invariant of the left Cauchy-Green tensor. This form will give a more accurate fit to the experimental data. It uses a strain energy function $\mathrm{W}$, whose derivative with respect to a strain component determines the corresponding stress component. The form of the Mooney-Rivlin strain energy potential is[2]:

$$
\begin{aligned}
& \sigma_{i j}=\frac{\partial W}{\partial \varepsilon_{i j}} \\
& W=\sum_{\mathrm{i}, \mathrm{j}=0}^{\infty} C_{\mathrm{ij}}\left(I_{\mathrm{i}}-3\right)^{\mathrm{i}}\left(I_{\mathrm{j}}-3\right)^{\mathrm{j}}
\end{aligned}
$$

Where $\mathrm{W}$ is the strain energy per unit of reference volume; $\mathrm{I}_{\mathrm{i}}$ and $\mathrm{I}_{\mathrm{j}}$ are the strain invariants. $\mathrm{C}_{\mathrm{ij}}$ is the constant of the Mooney-Rivlin material model. Usually two Mooney-Rivlin parameters $\left(\mathrm{C}_{10}\right.$ and $\left.\mathrm{C}_{01}\right)$ are used to describe hyper-elastic rubber deformation.

$$
\begin{gathered}
W=C_{10}\left(I_{1}-3\right)+C_{01}\left(I_{2}-3\right)+\left(\frac{C_{10}}{2}+C_{01}\right)\left(\frac{1}{I_{3}^{2}}-1\right)+D\left(I_{3}-1\right)^{2} \\
D=\frac{C_{10}(5 \mathrm{v}-2)+C_{01}(11 \mathrm{v}-5)}{2(1-2 \mathrm{v})}
\end{gathered}
$$

In the emulation software ANSYS/LS-DYNA, with the parameters density, Poisson's ratio , $\mathrm{C}_{10}$ and $\mathrm{C}_{01}$ the model can be determined. In this simulation, the parameters are listed in Table 1.

Table 1 Money-Rivlin parameters

\begin{tabular}{|c|c|c|c|}
\hline DENS & NUXY & $\mathrm{C}_{10}$ & $\mathrm{C}_{01}$ \\
\hline $1.8 \mathrm{e}-3$ & 0.499 & $0.736 \mathrm{e} 6$ & $0.184 \mathrm{e} 6$ \\
\hline
\end{tabular}

\section{Simulation}

Finite element analysis modeling. Figure. 1 shows the feature of the shape and geometric dimensions of soft punch stamping. In order from top to bottom, rigid mold that is rigid punch, soft mold material, metal sheet and rigid die are sketched of the stamping process.

In the forming process, the sheet metal placed between the soft punch and rigid die is filled into the rigid die by the soft mold material, which is deformed under the increasing force from the upper rigid die, until the sheet and the rigid die fit completely.

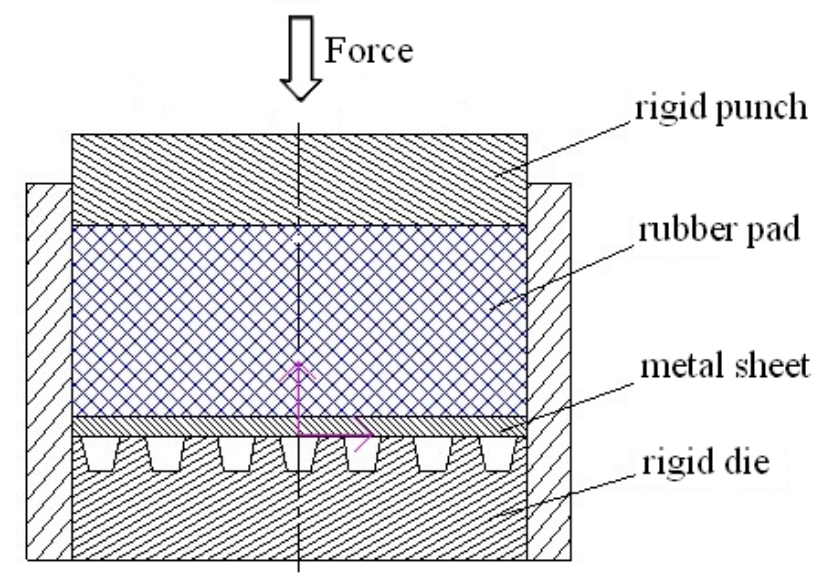

Fig.1 Sketch of soft punch stamping process

It's clear that every micro channel is independent under symmetrical loading during the forming process.

In the LS-DYNA software, a one channel finite element analysis model is set up based on the forming process(Figure 2a). The meshed model is shown in Figure 2b, Free mesh is used in the rigid die, mapped mesh of the rest, of which the element size are not much different. To reduce the 
computational design without affecting the result, the size of the rigid mold which is not the main deformed part is designed slightly larger.

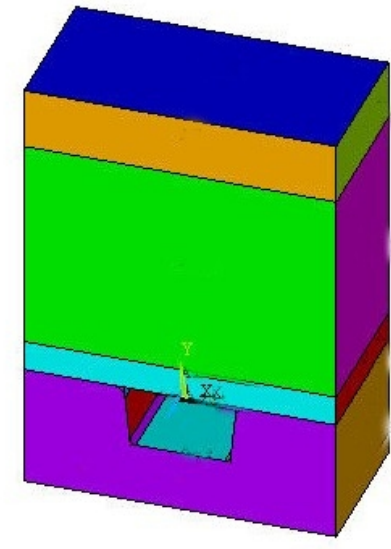

(a)One channel FEA model

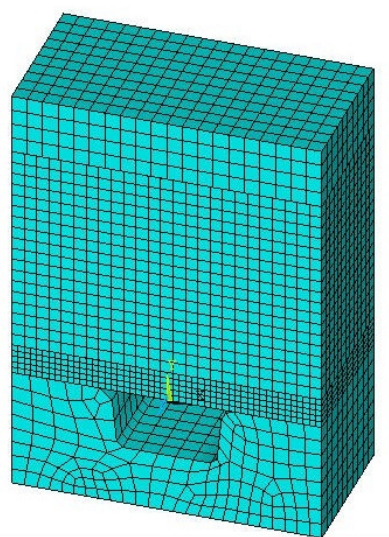

(b)One channel FEA mesh model

Fig.2 FEA model of one channel

Friction condition. Different from the traditional forming process, there are two friction interfaces: sheet with rigid die and sheet with soft punch.

Typically, this forming process uses surface contact model, namely Surface to Surface, in which is the main surface and secondary surface are involved. The harder one is selected as the main surface while the softer one the secondary surface, for it's determined based on the hardness.

In soft punch stamping process, there are three contact models: the rigid punch and soft mold, soft mold and sheet metal, sheet metal and upper rigid die. According to the former surface criterion, for that the soft material is the softest one, the main surface should be rigid punch and sheet metal in the first two molds. While compared with the sheet metal, rigid die is much harder, so its main surface. The correct definition can prevent penetration during the simulation.

Friction model is set to classical Coulomb model between the surface so only tangential friction of the sheet is taken into consideration and the equivalent friction coefficient varying based on the analysis conditions. Equivalent friction coefficient is not considered in this simulation for the main point is the feasibility.

Boundary conditions. Boundary conditions are set according to the practical experience or operating condition. Rigid punch, which is mainly used to load bearing strength, moves along the Y-axis, thus constraining a rigid punch all degrees of freedom except in the Y direction. Soft punch and sheet set are the same as the rigid punch. For the rigid die, its all degrees of freedom should be constrained to avoid unexpected problems.

In the analysis of rigid mold stamping, punch is set time - displacement load to complete the stamping process. While in the soft punch stamping model, this load is difficult to obtain the correct result, because the soft mold material is incompressible hyperelastic material, not having applied load displacement hydrostatic pressure already exists.

With it, little displacement will cause a lot of changes, so we apply load in this model instead of time - displacement load which don't suit for the material that is sensitive to the displacement. Studies have shown the relationship between the punch pressure and the channel depth, as well as the height-diameter ratio, according to which we set the punch load $12 \mathrm{KN}$.Under this circumstance , the material can fill up the mode to fit the rigid die and get the expected shape.

\section{Simulation results and discussions}

The soft punch stamping process is divided into three stages: the first stage is the deformation of soft punches with the press of upper rigid die; the second stage is metal sheets' deformation under the 
press of soft punches to reach the bottom of rigid die; the last stage is the filling of the metal sheets until it fits the rigid die.

Seen in the soft mold pressing, the entire stamping process is more than a soft-rigid mold stamping mold deformation stage, it would cause the entire stamping analysis time increases a lot.

Figure 3 is the simulation results of the second stage in soft punch process. It's clear to see from the graph that the maximum stress is $56332 \mathrm{~Pa}$ and the stress distribution in the rest part is relativity even. Yet, there is stress concentration at the bell of rigid die.

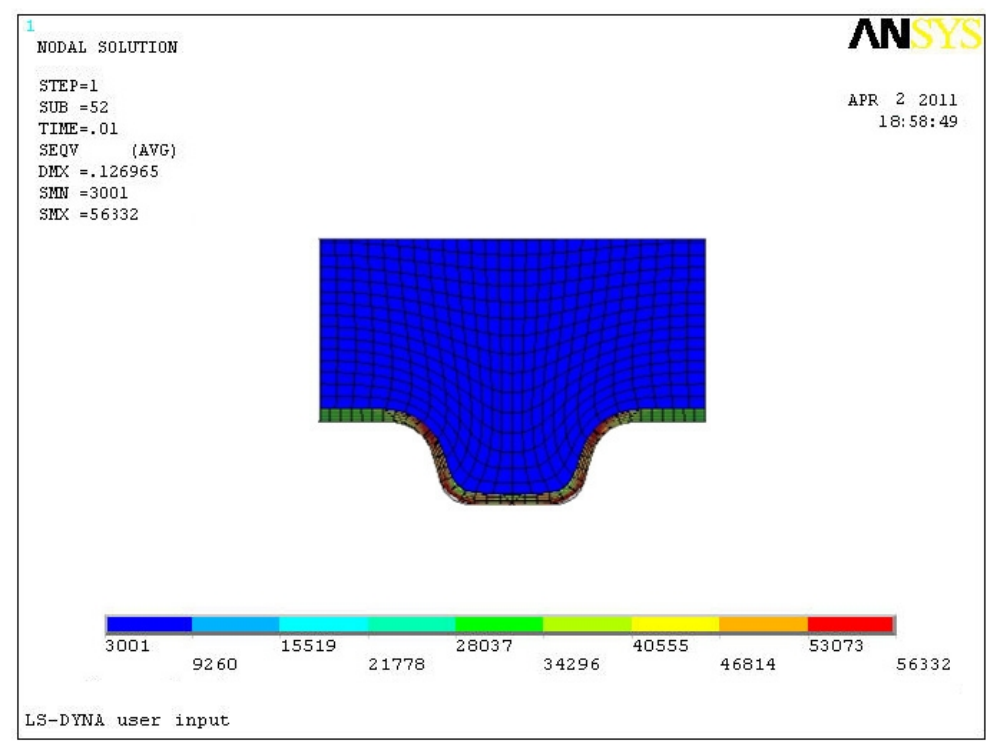

Fig.3 Stress distribution of formed sheet by soft punch stamping process

\section{Conclusion}

Soft punch stamping process is studied in this paper by numerical simulations. Forming process numerical simulations show that the micro channel parts are very unevenly deformed and the formed parts can be divided into deformed region and undeformed region. It also can be found that the defect in this forming process is the crack because of stress concentration at the bell of rigid die in the second stage. So in practical production, we should select different parameters for different materials to get the high quality workpiece.

\section{References}

[1] D.J. Browne, E. Battikha, Optimisation of aluminium sheet forming using a flexible die, J. Mater. Process. Technol. 55 (1995) 218-223.

[2] M.H. Dirikolu, E. Akdemir, Computer aided modelling of flexible forming process, J. Mater. Process. Technol. 148 (2004) 376-381.

[3] S. Thiruvarudchelvan, The potential role of flexible tools in metal forming, J. Mater. Process. Technol. 122 (2002) 293-300.

[4] M. Ramezani, Z.M. Ripin, R. Ahmad, Computer aided modelling of friction in rubber-pad forming process, J. Mater. Process. Technol. 209 (2009) 4925-4934.

[5] L.Peng, P. Hu, X. Lai, D. Mei, J. Ni, Investigation of Micro/Meso Sheet Soft Punch Stamping Process-Simulation and Experiments, Mater. Des., 30(2009) 783-790. 